\title{
Clathrin is essential for meiotic spindle function in oocytes
}

\author{
Jurriaan J Hölzenspies ${ }^{1,2}$, Bernard A J Roelen ${ }^{1}$, Ben Colenbrander ${ }^{1}$, Roland A P Romijn ${ }^{3}$, \\ Wieger Hemrika ${ }^{3}$, Willem Stoorvogel ${ }^{2}$ and Theo van Haeften ${ }^{2}$ \\ Departments of ${ }^{1}$ Farm Animal Health and ${ }^{2}$ Biochemistry and Cell Biology, Faculty of Veterinary Medicine, \\ Utrecht University, 3584 CM Utrecht, The Netherlands and ${ }^{3}$ U-Protein Express BV, 3584 CH Utrecht, The Netherlands \\ Correspondence should be addressed to T van Haeften; Email: t.vanhaeften@uu.nl
}

\begin{abstract}
In the mammalian ovary, oocytes are arrested at prophase of meiosis I until a hormonal stimulus triggers resumption of meiosis. During the subsequent meiotic maturation process, which includes completion of the first meiotic division and formation of the second metaphase spindle, oocytes acquire competence for fertilization. Recently, it was shown that clathrin, a cytosolic protein complex originally defined for its role in intracellular membrane traffic, is also involved in the stabilization of kinetochore fibers in mitotic spindles of dividing somatic cells. However, whether clathrin has a similar function in meiotic spindles in oocytes has not been investigated previously. Our results show that endogenous clathrin associates with the meiotic spindles in oocytes. To study the function of clathrin during meiotic maturation, we microinjected green fluorescent protein-tagged C-terminal and N-terminal dominant-negative clathrin protein constructs into isolated porcine oocytes prior to in vitro maturation. Both protein constructs associated with meiotic spindles similar to endogenous clathrin, but induced misalignment and clumping of chromosomes, occurrence of cytoplasmic chromatin and failure of polar body extrusion. These data demonstrate that clathrin plays a crucial role in meiotic spindle function in maturing oocytes, possibly through spindle stabilization.

Reproduction (2010) 140 223-233
\end{abstract}

\section{Introduction}

During development, mammalian oocytes are arrested at prophase I, and resume meiosis when maturation is initiated in response to the luteinizing hormone surge. Oocyte maturation is characterized by several key events, including disintegration of the nuclear envelope, also referred to as germinal vesicle breakdown (GVBD), formation of the first metaphase spindle, extrusion of the first polar body, and formation of the second metaphase spindle. In most mammals, oocytes enter a second period of meiotic arrest at metaphase II (MII), which is maintained until fertilization (Mehlmann 2005b, Richard 2007). Nondisjunction of chromosomes during the meiotic divisions of oocytes is a major source of genetic disorders in humans (Martin 2008), and it is thus important to understand the machinery that controls proper chromosome segregation. In mitotic cells, the kinetochore fibers of the metaphase spindle are associated with and stabilized by a large cytosolic protein, clathrin (Royle et al. 2005, Royle \& Lagnado 2006).

Clathrin is a ubiquitously expressed protein, and has a prominent role in intracellular membrane transport pathways that rely on clathrin-coated vesicles (Kirchhausen 2000, Okamoto et al. 2000, Royle et al. 2005, Ungewickell \& Hinrichsen 2007). Clathrin forms a large three-legged hexameric protein complex called triskelion, which is composed of three heterodimers of a $\sim 190 \mathrm{kDa}$ heavy chain (CHC) and a $\sim 25 \mathrm{kDa}$ light chain (CLC). Triskelions can polymerize into a polyhedral coat that drives the formation of clathrin-coated vesicles, which bud off from several membranes, including the plasma membrane and the trans-Golgi network (Kirchhausen 2000, Ungewickell \& Hinrichsen 2007). A less well-known function of clathrin, independently of its role in membrane transport, is to prevent misalignment and mis-segregation of chromosomes in mitotic cells, presumably by bridging microtubules (Royle et al. 2005, Royle \& Lagnado 2006). Clathrin-depleted somatic cells show diverse mitotic defects, including the activation of the spindle checkpoint and disruption of cytokinesis, culminating in aberrant chromosome segregation and incomplete cell division. Although clathrin has also been observed to associate with the second meiotic spindle in mouse oocytes (Maro et al. 1985), a role for clathrin in spindle function in maturing oocytes has not been established. Mammalian oocytes form spindles that are generally much larger than mitotic spindles, and arrest at MII after in vitro maturation, thus providing ideal conditions to study the spindle function of clathrin.

In the present study, we have investigated the role of clathrin in the meiotic progression of maturing porcine oocytes. Clathrin function at metaphase spindles 
requires both the $\mathrm{N}$ - and $\mathrm{C}$-terminal domains of $\mathrm{CHC}$ in somatic cells (Royle et al. 2005, Royle \& Lagnado 2006, Yamauchi et al. 2008). To interfere with clathrin function, we microinjected green fluorescent protein (GFP)-tagged constructs that constituted either the $\mathrm{N}$ - or the C-terminal domain of porcine $\mathrm{CHC}$. Our data provide the first insight into the involvement of clathrin in mammalian oocyte maturation and demonstrate that clathrin is essential for meiotic spindle function.

\section{Results \\ Distribution of endogenous clathrin during oocyte maturation}

To investigate the distribution of endogenous clathrin, porcine oocytes were fixed either directly after isolation or after $44 \mathrm{~h}$ of in vitro maturation, immunolabeled for heavy chain clathrin and analysed using confocal laser scanning microscopy. All freshly isolated oocytes were at the GV stage, whereas nearly all $44 \mathrm{~h}$ matured oocytes reached the MII stage. In both immature GV and MII stage oocytes, $\mathrm{CHC}$ was distributed throughout the cytoplasm, but predominantly in association with punctate structures at the oocyte cortex, possibly corresponding to clathrincoated vesicles (Fig. $1 \mathrm{~A}$ and C). In MII stage oocytes, CHC was also observed at the metaphase spindle and in the polar body (Fig. 1C). Oocytes labeled for CLC showed similar patterns for both stages (data not shown). Control oocytes labeled with identical concentrations of negative control mouse IgG showed no staining at identical confocal settings (Fig. 1B and D), demonstrating the specificity of clathrin labeling. Notably, staining for $\mathrm{CHC}$ was relatively low in the surrounding cumulus cells, both at the onset of maturation and at MII arrest (Fig. 1A and $\mathrm{C}$ ). The presence of clathrin on microtubules at the meiotic spindle was confirmed by immuno-double labeling $44 \mathrm{~h}$ matured oocytes for $\mathrm{CHC}$ and tubulin (Fig. 1E-H). To confirm that $\mathrm{CHC}$ associated with spindle fibers, $43 \mathrm{~h}$ matured oocytes were exposed to $10 \mu \mathrm{M}$ nocodazole for $1 \mathrm{~h}$ to depolymerize the microtubules
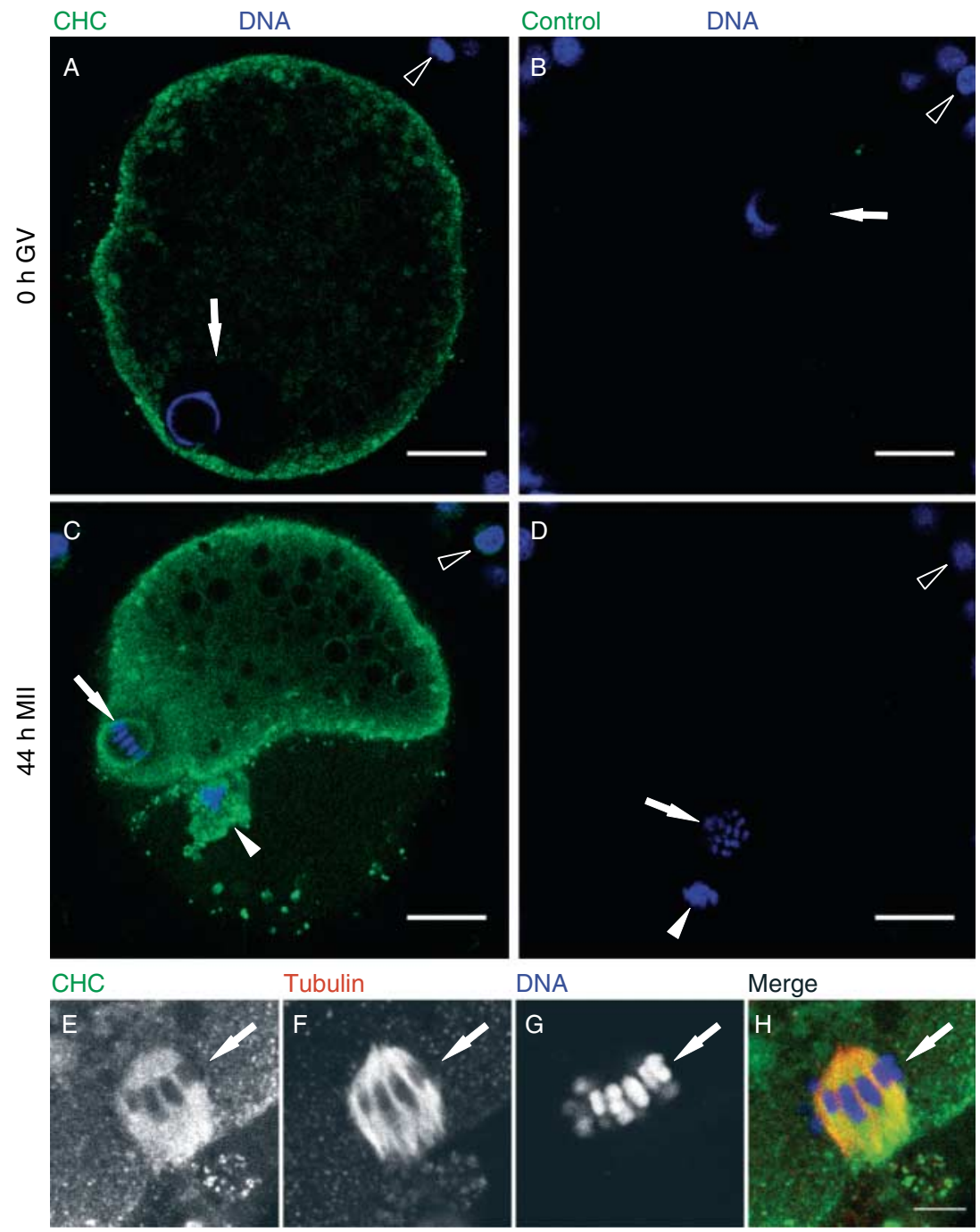

Figure 1 Distribution of endogenous clathrin during oocyte maturation. (A and B) $0 \mathrm{~h} \mathrm{GV}$ stage oocytes labeled for DNA (blue) and $\mathrm{CHC}$ (A; green), or irrelevant control antibody (B; green). Arrows indicate the GV. (C and D) $44 \mathrm{~h}$ MII stage oocytes labeled for DNA (blue) and $\mathrm{CHC}$ (C; green), or irrelevant control antibody (D; green). Arrows and arrowheads indicate the MII spindle and the polar body respectively. Open arrowheads indicate cumulus cells that remained associated with the zona pellucida (not stained) in all images. Please note that the clathrin-stained oocytes in this figure show mild (GV stage) to severe (MII stage) denting of the plasma membrane, and this difference in fixation effects is presumably caused by the loss of gap junctions between the oocyte and the transzonal projections from the cumulus cells. The resulting morphology may appear to be aberrant, but is in fact normal for oocytes at these stages. Scale bars, $20 \mu \mathrm{m}$. (E-H) Single confocal section at an MII spindle of a $44 \mathrm{~h}$ matured oocyte labeled for $\mathrm{CHC}$ ( $\mathrm{E}$ and green in $\mathrm{H}$ ), tubulin ( $\mathrm{F}$ and red in $\mathrm{H}$ ), and DNA ( $\mathrm{G}$ and blue in $\mathrm{H}$ ), showing colocalization (yellow) of $\mathrm{CHC}$ and tubulin in the merge $(\mathrm{H})$. Arrows indicate the metaphase plate. Scale bar, $10 \mu \mathrm{m}$. 
that make up the spindle. Nocodazole-treated oocytes lacked both tubulin and clathrin labeling around the DNA in oocytes that showed an extruded polar body, whereas these labels were present around the DNA in DMSO-treated controls and nocodazole-treated oocytes that were allowed to recover for $1 \mathrm{~h}$ (Supplementary Figure 1, see section on supplementary data given at the end of this article). Collectively, these data demonstrate that the association of clathrin with the spindle relies on the presence of intact microtubules.

\section{Clathrin constructs}

To study clathrin function during oocyte maturation, truncated dominant-negative GFP-tagged $\mathrm{CHC}$ protein constructs were first generated using transfected HEK293E cells and isolated to homogeneity (see Materials and Methods section and Fig. 2A). The C-terminal construct (GFP-C-CHC) contained the $\mathrm{CHC}$ trimerization domain, the CLC-binding domain, and GFP- and His-tags at its $\mathrm{N}$-terminus. A similar construct was previously shown to trimerize with endogenous clathrin and inhibit clathrin-mediated endocytosis in HeLa cells (Liu et al. 1998). Since trimerization of clathrin is also important for its function on spindles in somatic cells (Royle \& Lagnado 2006), GFP-C-CHC was expected to interfere with the spindle function of clathrin in oocytes. The N-terminal construct (N-CHC-GFP) encompassed a domain that was previously demonstrated to interfere with clathrin-mediated endocytosis and to associate with mitotic spindles in somatic cells (Royle et al. 2005), and contained GFP- and His-tags at its C-terminus. We also tried to produce these dominant-negative $\mathrm{CHC}$ protein constructs with GFPtags on the other side and a GFP-tagged full-length $\mathrm{CHC}$ construct. Unfortunately, these constructs were not stably expressed by our producer cell line. Instead, non-conjugated GFP was used as a negative control.

Isolated constructs were then microinjected into immature oocytes. To control for the amount of injected protein, total GFP fluorescence in oocytes was measured by epifluorescence microscopy (Lee 1989) directly after injection (Fig. 2B). Of successfully injected oocytes, total fluorescence did not differ significantly between oocytes injected with GFP or either one of the $\mathrm{CHC}$ constructs (Fig. 2C). After injection, oocytes were allowed to mature for $44 \mathrm{~h}$, fixed, immunolabeled for tubulin, and stained for DNA. Consistent with the distribution of endogenous clathrin, both N-CHC-GFP and GFP-C-CHC accumulated at the meiotic spindle (Fig. 3A and B). To quantify recruitment of CHC constructs to the meiotic spindles (Royle et al. 2005), we determined GFP fluorescence intensities in representative areas on the spindle relative to the oocyte cortex (Fig. 3A-C). Both N-CHC-GFP and GFP-C-CHC were significantly enriched on the spindle in comparison with non-conjugated GFP (Fig. 3D).

\section{Clathrin constructs disrupt meiotic divisions in oocytes}

Our next step was to investigate the effects of injected $\mathrm{N}-\mathrm{CHC}-\mathrm{GFP}$ and GFP-C-CHC on oocyte maturation. A normal MII pattern was observed in the majority of oocytes that were injected with non-conjugated GFP
A
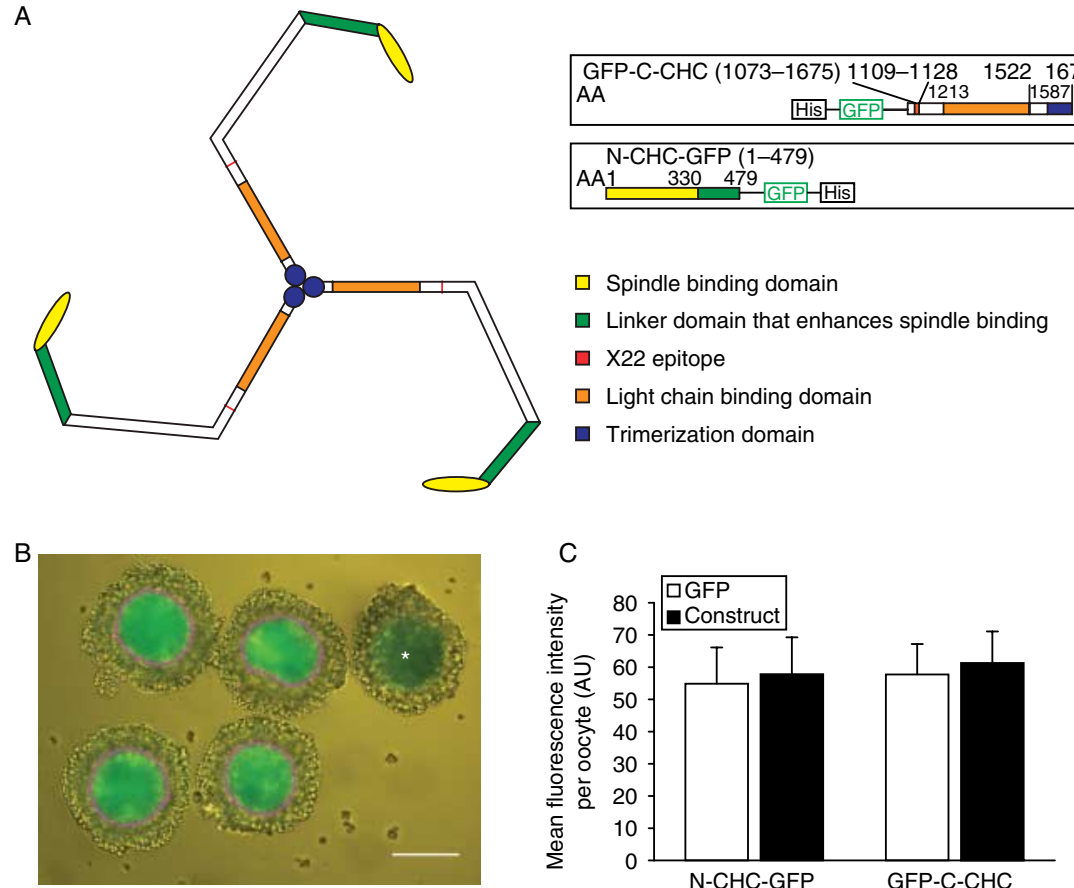

www.reproduction-online.org

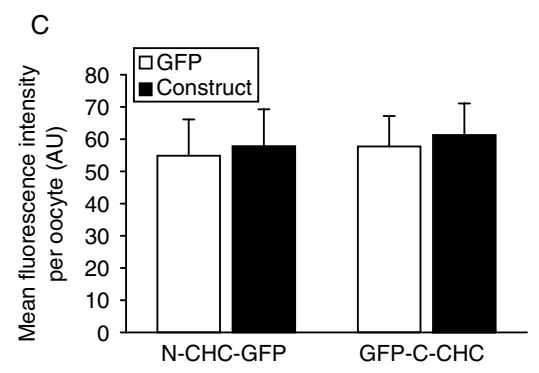

Figure 2 Clathrin constructs. (A) Schematic overview of the $\mathrm{CHC}$ constructs. The color-coded domains on the constructs are indicated in identical colors on the clathrin triskelion shown on the left, and the location of the GFP tag on each of the constructs is shown on the right. (B) Merged brightfield and fluorescent images showing five oocytes injected with GFP-C-CHC. As an example, the oocyte marked with * was removed from the experiment immediately after injection based on its relatively low fluorescence intensity. A region of interest (ROI; shown in magenta) was drawn around the remaining four oocytes by thresholding the fluorescent image (the same threshold was used for all injection experiments in this study). (C) Bar graph showing measured total fluorescence within threshold-generated ROIs for injected oocytes. Bars represent mean fluorescence intensity \pm s.D. in arbitrary units (AU) of 45-63 oocytes from three independent experiments. For the experiments in Figs 3-6, no significant difference in fluorescence intensity was found between oocytes injected with N-CHC-GFP $(P=0.24)$, GFP-C-CHC $(P=0.09)$, or GFP using a two-tailed homoscedastic Student's $t$-test. 

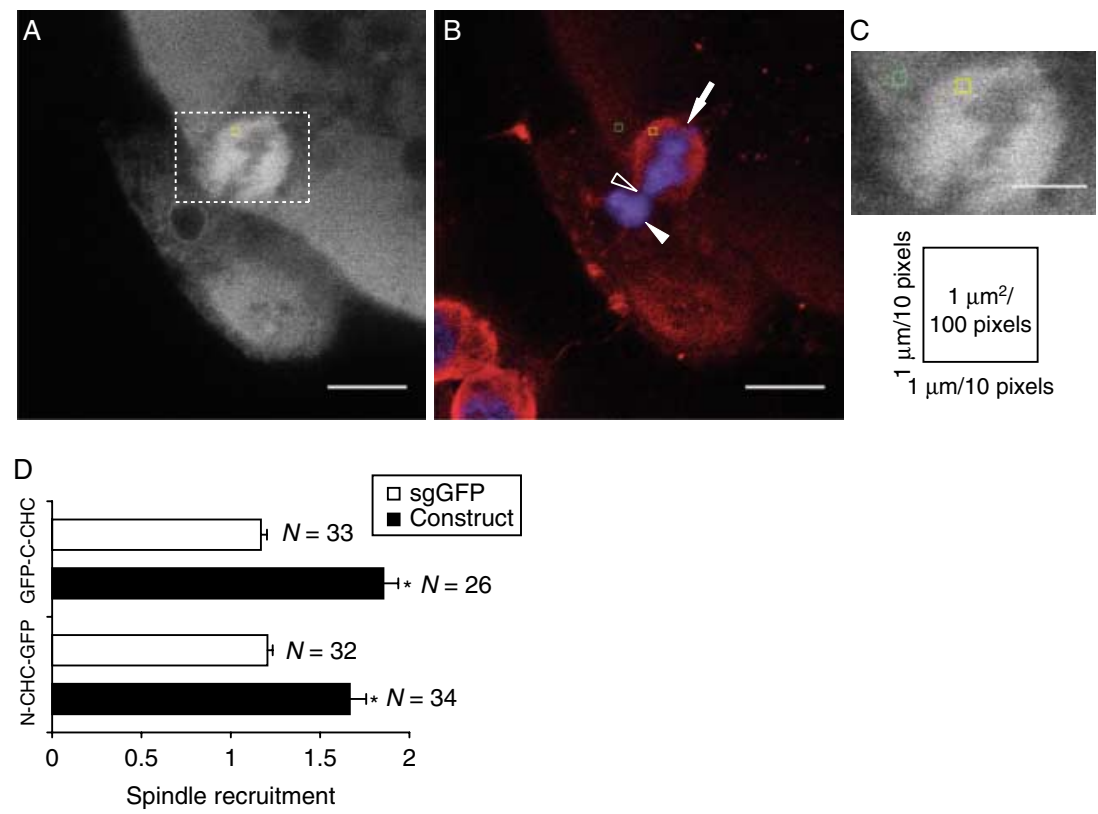

Figure 3 Clathrin constructs specifically localize to the meiotic spindle. (A) An oocyte showing the distribution of injected N-CHC-GFP. (B) The distributions of tubulin (red) and DNA (blue) in the same oocyte as in A, showing a typical phenotype observed in CHC construct-injected oocytes, which is characterized by clumping of the chromatin on the MII spindle (arrow) and an extruded polar body (arrowhead) that remained attached to the spindle via a 'DNA bridge' (open arrowhead). Scale bars, $20 \mu \mathrm{m}$. (C) Enlargement of the area indicated by the dashed rectangle in A. Scale bar, $5 \mu \mathrm{m}$. A schematic representation indicating the size of the regions of interest (ROIs) on the spindle (yellow square) and in the cortex (green square) is shown below the image. Spindle recruitment was calculated by dividing the mean GFP fluorescence intensity in the ROI on the spindle by that in the cortex. To calculate spindle recruitment, two ROIs on the spindle (one on each side of the metaphase plate) and two ROls in the cortex were analysed for each oocyte. To ensure objective measurements, each spindle/cortex-pair of ROls was created within a single confocal section showing only tubulin and DNA staining (as in B) and transposed onto the equivalent section showing GFP fluorescence (as in A). (D) Bar chart showing spindle recruitment in oocytes injected with control GFP or $\mathrm{CHC}$ constructs. Bars represent mean spindle recruitment \pm s.E.M. from three independent experiments, and the number of oocytes analysed $(\mathrm{N})$ is shown next to each bar. Both N-CHC-GFP $(P<0.001)$ and GFP-C-CHC $(P<0.001)$ revealed significantly higher recruitment to MII spindles (asterisks) compared with GFP in injected oocytes using a two-tailed heteroscedastic Student's $t$-test.

(Figs $4 \mathrm{~A}-\mathrm{D}$ and $5 \mathrm{~A}$ and $\mathrm{B}$ ). Severe defects were observed, however, in both N-CHC-GFP- and GFP-C-CHCinjected oocytes. Both $\mathrm{CHC}$ constructs caused misalignment of chromosomes on the metaphase plate and formation of large clumps of condensed chromatin interspersed with patches of less condensed chromatin (Fig. 4E-T). In addition, chromosomes were regularly observed outside the metaphase spindle (e.g. open arrowhead in Fig. 4Q-T), and the polar body often remained connected to the oocyte via a 'DNA bridge' (open arrowheads in Fig. 4E-P) that varied in thickness and length between the injected oocytes (short and thin in Fig. 4E-H, short and thick in Fig. $4 \mathrm{I}-\mathrm{L}$, and long and thin in Fig. 4M-P). These bridges displayed no tubulin labeling, indicating that the spindle midbody was no longer present. These phenotypes are illustrated more clearly in Supplementary Movies 1-4 (see section on supplementary data given at the end of this article) which show $360^{\circ}$ rotations of $3 \mathrm{D}$ reconstructions of the same oocytes as in Fig. 4A-H. In a subset of GFP-C-CHCinjected oocytes, condensed chromosomes were scattered throughout the area around the metaphase spindle. These scattered chromosomes formed small clusters that also contained tubulin (open arrowheads in Fig. 4Q-T) creating small spindle-like structures. Since defects at MII may result from abnormal progression through meiosis I (MI), oocytes that were arrested at $\mathrm{Ml}$ after $44 \mathrm{~h}$ of maturation were also examined in detail. Typical aberrant $\mathrm{MI}$ morphology observed in these constructinjected oocytes included incomplete condensation and clumping of chromosomes (Fig. 6), abnormally dense astral microtubule networks (Fig. 6E-H), loose chromosomes outside the metaphase plate (filled arrowheads in Fig. 6), and tubulin fibers that connected the spindle to the loose chromosomes (open arrowheads in Fig. 6I-P).

To quantify the effects of the $\mathrm{CHC}$ constructs on oocyte maturation, $44 \mathrm{~h}$ matured oocytes were scored into four categories based on their tubulin and DNA staining patterns: aberrant GV (clearly visible nucleus), aberrant MI (with oocytes from prometaphase I up to and including telophase I), aberrant MII (clearly visible metaphase spindle and polar body, showing abnormal structure), and normal MII (clearly visible metaphase spindle and polar body). Oocytes that showed a normal $\mathrm{GV}$ or $\mathrm{MI}$ pattern after maturation were also scored as aberrant, since these oocytes failed to complete 


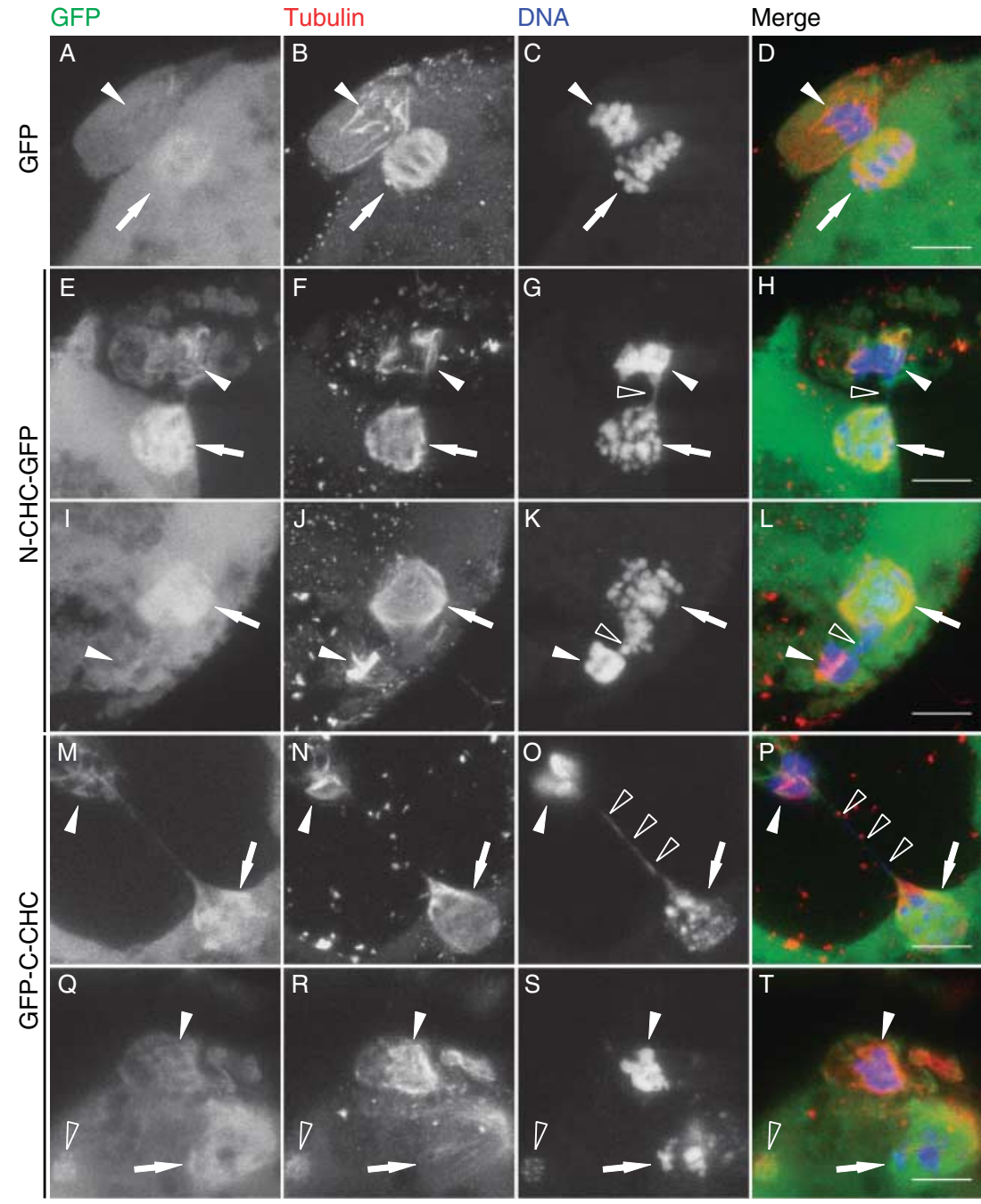

Figure 4 Aberrant MII profiles generated in response to clathrin constructs. Oocytes were injected with GFP or GFP-CHC constructs (left column and green in the merged fourth column), matured for $44 \mathrm{~h}$, fixed, and labeled for the presence of tubulin (second column and red in the merged fourth column) and DNA (third column and blue in the merged fourth column). (A-D) Control GFP-injected oocyte showing a normal MII spindle and polar body. (E-L) Two examples of N-CHC-GFP-injected oocytes showing patchy condensation of chromosomes on the MII spindle and a 'DNA bridge' (open arrowheads) between the spindle and the polar body. (M-T) Two examples of GFP-C-CHCinjected oocytes showing either a long DNA bridge (open arrowheads in $\mathrm{M}-\mathrm{P}$ ), or misaligned chromosomes on the metaphase spindle (arrow in Q-T) and cytoplasmic chromatin (open arrowheads in Q-T). Filled arrows and arrowheads indicate the position of the MII spindle and polar body respectively. Scale bars, $10 \mu \mathrm{m}$. maturation. Oocytes that could not be scored into any of these categories $(\sim 4 \%)$ were excluded from the analysis. Of the non-injected and GFP-injected oocytes, $60-70 \%$ showed normal morphology at MII (Fig. 5A and B). In contrast, of the N-CHC-GFP- and GFP-C-CHC-injected oocytes, only 13 and $2 \%$ respectively reached normal MII, while most showed an aberrant MII morphology. Statistical analysis of these data shows that oocytes injected with N-CHC-GFP or GFP-C-CHC were significantly inhibited in progressing through maturation as compared with GFP-injected oocytes. In both N-CHCGFP and GFP-C-CHC conditions, the strongest significant effect was found for the ratio of aberrant versus normal MII oocytes compared with that in the control group. The ratio of aberrant MI versus normal MII oocytes was, however, also significantly higher for both $\mathrm{CHC}$ constructs. Only GFP-C-CHC-injected oocytes differed significantly in comparison with the control group in the ratio of aberrant GV versus normal MII phenotypes. A summary of these results and their significance is shown in Fig. 5C.

To specify MII aberrancies in more detail, we scored aberrant MII oocytes by phenotype using the following categories: misaligned chromosomes (chromatin outside the equator of the MII spindle), chromosome clumps (large clumps of condensed chromatin interspersed with patches of less condensed chromatin), DNA bridge (direct link between chromatin in the polar body and the oocyte) and cytoplasmic chromatin (loose chromosomes present in the cytoplasm). Almost all oocytes injected with either one of the $\mathrm{CHC}$ constructs showed misaligned chromosomes and chromosome clumps, whereas DNA bridges and cytoplasmic chromatin were observed in only $20-45 \%$ of oocytes. Statistical analysis of these results using $\chi^{2}$ tests did not reveal any significant differences between $\mathrm{N}$-CHC-GFP- and GFP-C-CHC-injected oocytes, suggesting that the effects of these constructs on oocyte maturation were identical (Fig. 5D). 

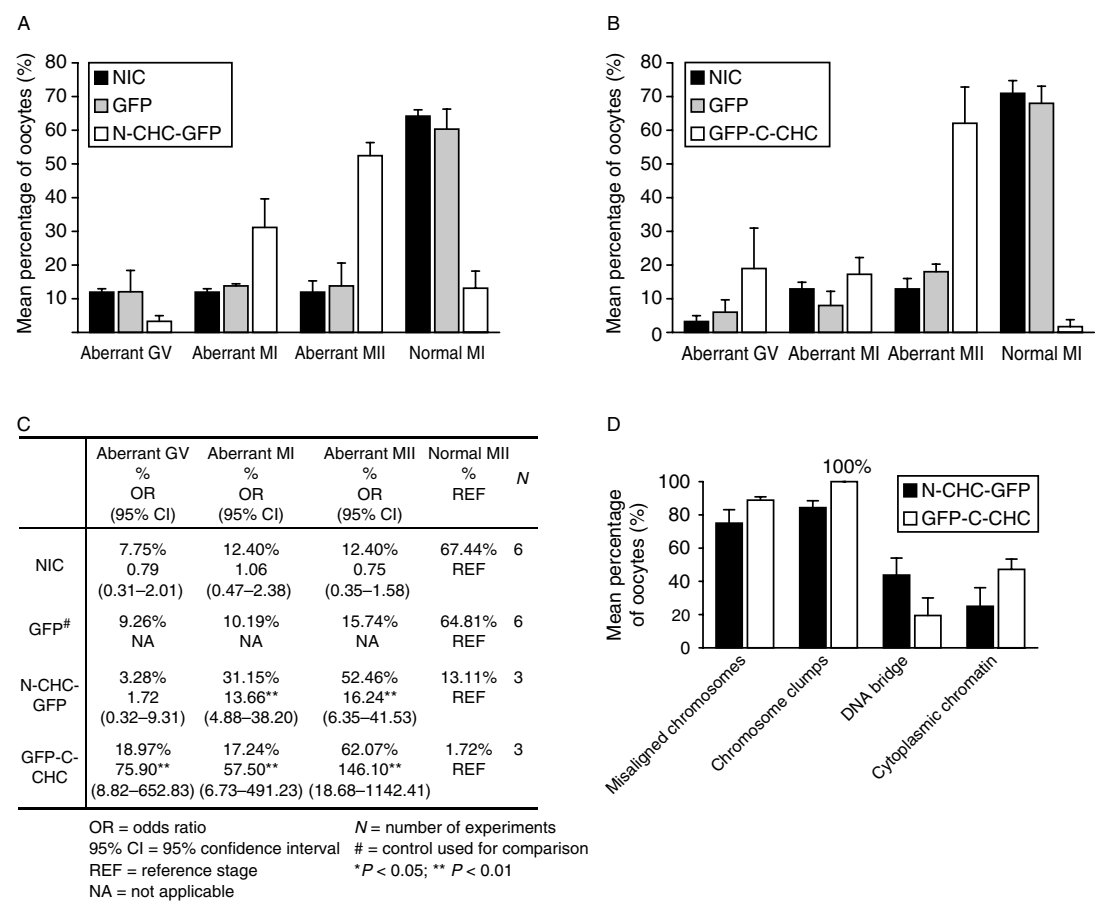

D

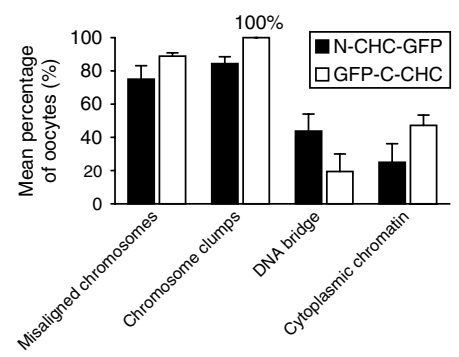

Figure 5 Quantification of aberrant meiotic profiles after injection of clathrin constructs. (A and B) Bar graphs showing percentages of 44 h matured oocytes at various stages after injection as determined in Fig. 4. Bars represent weighted mean percentage \pm weighted S.E.M. $(N=3$ independent experiments) of oocytes at the indicated stages. The indicated conditions are non-injected control oocytes (NIC; 67 and 62 oocytes in $\mathrm{A}$ and $\mathrm{B}$ respectively), GFP-injected oocytes (GFP; 58 and 50 oocytes in A and B respectively), N-CHC-GFP-injected oocytes (N-CHC-GFP; A; 61 oocytes), and GFP-C-CHC-injected oocytes (GFP-C-CHC; B; 58 oocytes). Significance of the results is indicated in the results section. (C) Table showing a summary of the analysis of the data shown in $(\mathrm{A})$ and $(\mathrm{B})$. The ratio of oocytes at each aberrant stage versus oocytes at the reference stage (normal MII) was compared between NIC/N-CHC-GFP/GFP-C-CHC and GFP conditions using multinomial logistic regression models. Several models were employed to investigate the interaction between maturation stage, which was used as an ordinal variable, and condition. Based on a minimized Akaike information criterion (AIC) value, a model that incorporated both N-CHC-GFPand GFP-C-CHC conditions and their respective controls, and used experiment number to control for random effects was chosen for final analysis. Significance of the results is indicated by asterisks $(P<0.01)$. (D) Aberrant MII oocytes in (A) and (B) were scored for observed defects and analysed by $\chi^{2}$ tests, which did not reveal any significant differences between N-CHC-GFP- and GFP-C-CHC-injected oocytes $(P>0.05$; Bonferroni corrected).

\section{Discussion}

In this study, we have demonstrated that clathrin is associated with meiotic spindles in porcine oocytes, and that interference with clathrin function by injection of dominant-negative $\mathrm{CHC}$ protein constructs disrupted meiosis progression. Moreover, our results support the notion that clathrin is involved in chromosome segregation during oocyte meiosis.

Clathrin is a cytosolic protein that, in addition to its role in membrane transport, was recently shown to stabilize the mitotic spindle in somatic cells (Royle et al. 2005, Royle \& Lagnado 2006). This role for clathrin in mitosis was later questioned for a chicken pre-B lymphoma cell line (DKO-R). Although the localization of clathrin at the spindle during mitosis is highly consistent between different cell lines, clathrin-depleted DKO-R cells did not show mitotic defects (Royle et al. 2005, Borlido et al. 2008). These data suggest the existence of cell type-specific differences in spindleassociated clathrin function. It should be noted, however, that DKO-R cells were specifically developed to study clathrin function while preventing apoptosis caused by clathrin depletion, and could have acquired adaptations that attenuate the mitotic phenotype of clathrin depletion that was observed in other cell lines (Wettey et al. 2002, Royle et al. 2005, Borlido et al. 2008). Although the necessity of clathrin function on mitotic spindles may vary between different cell lines, a conserved role for clathrin in stabilizing meiotic spindles is plausible, given that spindle instability is closely linked to aneuploidy (Jones 2008). Although clathrin also serves as an important factor in intracellular membrane transport (Kirchhausen 2000, Ungewickell \& Hinrichsen 2007), the potential functions of clathrin in oocytes have not been investigated previously. Consistent with previous observations for mouse MII oocytes (Maro et al. 1985), we demonstrate by immunofluorescence microscopy that clathrin associates with metaphase spindles in porcine oocytes (Fig. 1). The initial observations by Maro et al. (1985) were thought to reflect association of clathrin-coated vesicles with spindle fibers. In somatic cells, however, clathrin was shown to bind the mitotic spindle directly, while membranes did 


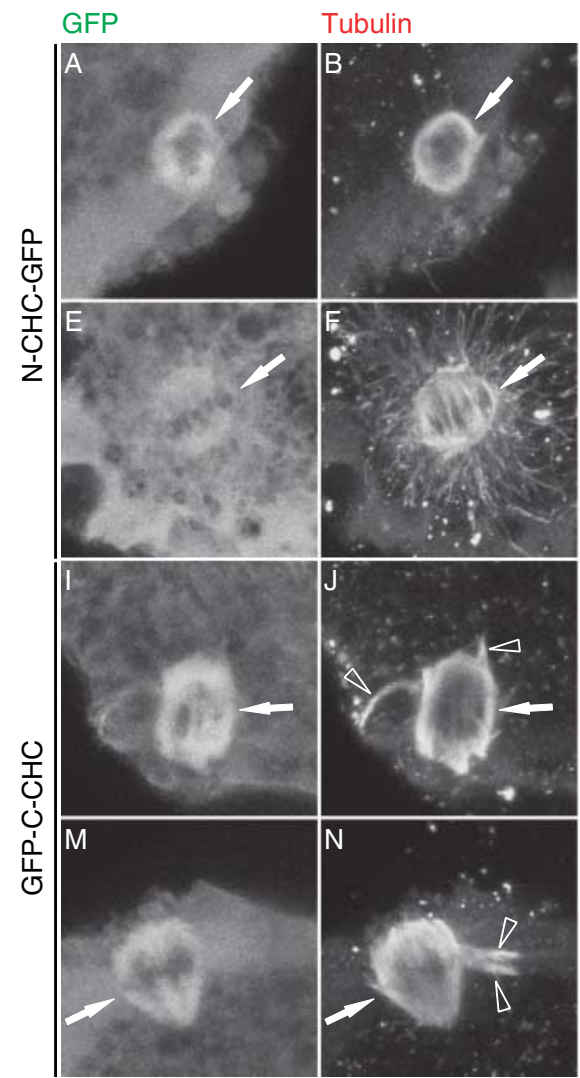

DNA

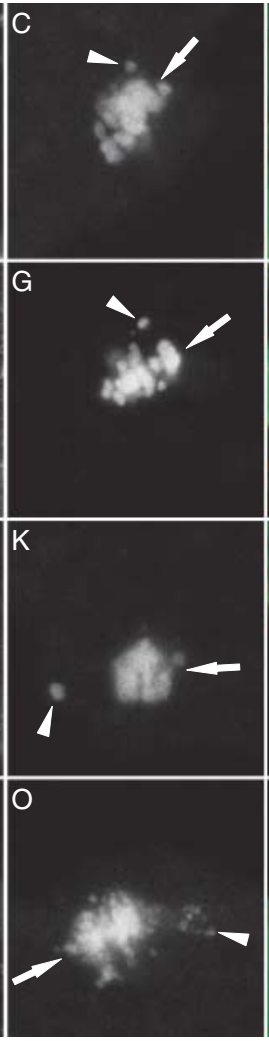

Merge

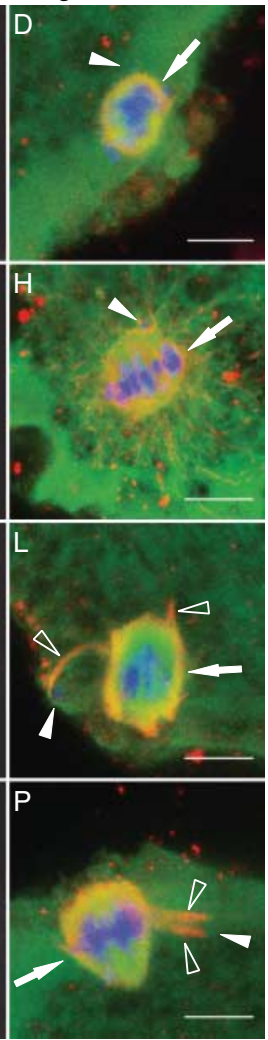

Figure 6 Aberrant $\mathrm{MI}$ profiles in response to clathrin constructs. Labeling and imaging of oocytes were performed as in Fig. 4. Examples of $\mathrm{N}$-CHC-GFP-injected (A-H) and GFP-C-CHCinjected (I-P) oocytes arrested at $\mathrm{Ml}$ after $44 \mathrm{~h}$ of maturation are shown. These $\mathrm{Ml}$ arrested oocytes show clumping of chromosomes on the metaphase plate, abnormal spindle morphology and 'loose' chromosomes that have failed to align on the metaphase plate. Arrows indicate the metaphase plate, filled arrowheads indicate 'loose' chromosomes and open arrowheads indicate tubulin fibers that emanate from the spindle. Maximum intensity $Z$ projections of three consecutive confocal sections ( $2 \mu \mathrm{m}$ intervals) are shown. Scale bars, $10 \mu \mathrm{m}$. not associate with the spindle (Royle et al. 2005, Royle \& Lagnado 2006). Using lipophilic dyes, we could not detect association of membranes with the meiotic spindle in oocytes (data not shown). Moreover, clathrin did not persist at the spindle upon depolymerization of spindle microtubules using nocodazole, and was recovered together with spindle microtubules within $1 \mathrm{~h}$ of nocodazole removal (Supplementary Figure 1). These data suggest that clathrin does not associate with the spindle matrix, as nocodazole-mediated depolymerization of the spindle in mitotic cells was previously shown to leave the spindle matrix in place (Lince-Faria et al. 2009).

Unlike somatic cells, fully grown mammalian oocytes are characterized by repressed transcription (Motlik et al. 1984, Fair et al. 1996, Bouniol-Baly et al. 1999, De La Fuente \& Eppig 2001, Bjerregaard \& Maddox-Hyttel 2004), and oocytes thus cannot be manipulated by cDNA transfection. Given the ubiquitous and crucial functions of clathrin, clathrin knockout animals have not been generated, and conditional knockout systems for clathrin in oocytes are not available. RNAi knockdown of endogenous clathrin in oocytes is also extremely unlikely to succeed, because clathrin is one of the most abundant proteins in cells (Goud et al. 1985), and has a very long half-life (Acton \& Brodsky 1990). Indeed, even in highly active mitotic cells, CHC RNAi does not show an effect on the mitotic index until $48 \mathrm{~h}$ after transfection (Royle et al. 2005). Although exogenous proteins can be expressed by injection of mRNA in porcine oocytes, the efficiency of such injection procedures is low $(\sim 30 \%$ of injected oocytes showed expression during maturation), and expression levels vary extensively between oocytes (Ohashi et al. 2001). Instead, the method used here to microinject dominant-negative clathrin protein constructs was highly efficient ( $\sim 75 \%$ of injected oocytes showed sustained fluorescence), and allowed instantaneous introduction of a reproducible amount of exogenous protein into each oocyte (Fig. 2). These microinjected GFP-tagged constructs, corresponding to the $\mathrm{N}$ - or C-terminal domains of $\mathrm{CHC}$ (Fig. 2), were both recruited to MII spindles (Fig. 3). N-CHC-GFP, which contains the previously determined spindle-binding domain (Royle et al. 2005), was recruited to the meiotic spindle to the same extent as GFP-C-CHC (Fig. 3). These observations suggest that both constructs contain spindle recruitment domains, although it cannot be excluded that our constructs have been recruited to the spindle by associated endogenous full-length $\mathrm{CHC}$ that may have been incorporated in 'mixed' triskelions. A recent study showed that formation of a complex, which consists of clathrin, B-Myb, and filamin, is required for recruitment of clathrin to the mitotic spindle in somatic cells, and the authors suggested that the C-terminal trimerization 
domain of clathrin could also be involved in the formation of this complex (Yamauchi et al. 2008). In another study, the N-terminal domain appeared to be indispensable for spindle localization, since a construct lacking this domain (331-1639) failed to be recruited to mitotic spindles (Royle \& Lagnado 2006). It should be noted that these results were obtained in somatic cells that had been depleted of endogenous clathrin by RNAi, and as such did not allow formation of mixed triskelions containing both endogenous $\mathrm{CHC}$ and the truncated $\mathrm{CHC}$ construct.

Oocytes injected with either one of our $\mathrm{CHC}$ constructs displayed severe defects in MII morphology (Figs 4 and 5) while maturation rates and MII morphology of GFP-injected control oocytes did not differ from those of non-injected controls (Figs 4A-D and $5 \mathrm{~A}$ and $\mathrm{B})$. Both $\mathrm{CHC}$ constructs caused misalignment of chromosomes and formation of chromosome clumps, which may be explained by $\mathrm{CHC}$ construct-induced failure of the spindle checkpoint during MI. When some homologous chromosomes separate normally and others remain attached to each other and are pulled either into the nascent polar body or into the oocyte, chromosome clumps would form on the second metaphase spindle. Moreover, the aberrant presence of homologous chromosome pairs on the second metaphase spindle may disrupt proper chromosome alignment. Indeed, oocytes that were arrested at $\mathrm{Ml}$ after $44 \mathrm{~h}$ of maturation as a consequence of the injection of $\mathrm{CHC}$ constructs showed congression failure (Fig. 6). In spite of this severe phenotype observed in some of the injected oocytes, the majority of oocytes that were injected with the $\mathrm{CHC}$ constructs did not arrest at $\mathrm{MI}$ (Fig. 5A and B), indicating failure of the $\mathrm{MI}$ spindle checkpoint. Although these inferences remain speculative, it is clear that future research on clathrin function in meiotic spindles should include investigation of checkpoint controls, particularly since clathrin adaptor proteins have been implicated in spindle function and checkpoint control in mitotic cells (Cayrol et al. 2002, Lehtonen et al. 2008).

Clathrin-mediated endocytosis may also have been affected by our CHC constructs. A possible effect of interference with endocytosis is failure of polar body cytokinesis, since it has been demonstrated that endocytosis at the cleavage furrow is important for cytokinesis in several types of mitotic cells (Niswonger \& O'Halloran 1997, Feng et al. 2002, Schweitzer et al. 2005, Albertson et al. 2005, Warner et al. 2006, Montagnac et al. 2008). These findings suggest that endocytosis may also be important for cytokinesis in oocytes. We observed that respectively 44 and $19 \%$ of $\mathrm{N}-\mathrm{CHC}-\mathrm{GFP}$ - or GFP-C-CHC-injected oocytes remained connected to their polar body via a DNA bridge (Figs 4E-P and 5D), demonstrating that chromosome segregation is incomplete in these oocytes. These cytoplasmic connections between oocytes and their polar bodies did not contain tubulin, suggesting that the spindle midbody, which may be involved in abscission during cytokinesis (Montagnac \& Chavrier 2008), was no longer present. These results are indicative of destabilization of the first meiotic spindle, given that cytokinesis is normally preceded by chromosome segregation. We conclude that interference with endocytosis-dependent cytokinesis cannot explain our results. Studies on Xenopus oocytes demonstrated that meiosis resumption involves endocytosis of a G-protein-coupled receptor (Wang \& Liu 2003, El-Jouni et al. 2007). The G-proteincoupled receptor GPR3 appears to be required for maintenance of meiotic arrest in mouse, rat, and human oocytes (Mehlmann et al. 2004, Hinckley et al. 2005, Mehlmann 2005a, DiLuigi et al. 2008), suggesting that endocytosis of this receptor may also be involved in resumption of oocyte meiosis in mammals. Since clathrin is required for endocytosis of specific receptors in somatic cells (Kirchhausen 2000, Moore et al. 2007, Ungewickell \& Hinrichsen 2007), these findings suggest an alternative role for clathrin in resumption of meiosis. Constructs similar to our $\mathrm{CHC}$ constructs have been demonstrated to interfere with membrane traffic in somatic cells (Liu et al. 1998, Royle et al. 2005). In our study, however, meiosis progression beyond GVBD was not affected in the majority of $\mathrm{CHC}$ construct-injected oocytes (Fig. 5A and B). Therefore, inhibition of G-protein-coupled receptor endocytosis cannot explain our results.

Taken together, our findings in porcine oocytes are consistent with a role for clathrin in stabilizing metaphase spindles (Royle et al. 2005, Royle \& Lagnado 2006). Disruption of clathrin function in maturing oocytes causes severe spindle-related defects, which may be due to spindle checkpoint failure and/or spindle destabilization during MI. Although we have established that clathrin plays a pivotal role in meiosis progression during porcine oocyte maturation, further research will be required to untangle the contributions of its functions to meiotic spindle stability.

\section{Materials and Methods}

\section{Recombinant clathrin constructs}

Porcine clathrin heavy chain was PCR amplified from porcine brain CDNA using the Expand Long Template PCR System (ELT-PCR; Roche Applied Science, Indianapolis, IN, USA) according to the manufacturer's instructions with primers designed based on homology between the known human (NM_004859), bovine (NM_174023), and mouse (NM_001003908) CHC sequences (Table 1, Primers for PCR), and sequenced (Baseclear, Leiden, The Netherlands; EMBL accession number FM210346, accession date 3 March 2009). The PCR product was then diluted 1:1000 and amplified again using Pfu DNA polymerase (Fermentas, Burlington, Ontario, Canada) to obtain coding cDNA for residues 1-479 ( $\mathrm{N}-\mathrm{CHC})$ and residues 1073-1675 (C-CHC) and to introduce in-frame BamHI - Notl restriction sites for C-terminal clathrin and 
Table 1 Primers for full-length, C-terminal and N-terminal clathrin heavy chain.

\begin{tabular}{|c|c|c|}
\hline Primers for PCR & Forward & Reverse \\
\hline Full-length clathrin + UTRs & $5^{\prime}$-GGA GGA GAC CAT ACC CCC CGA CAG-3' & 5'-AAC AGA TTG AAT ATT AAG CAG T-3' \\
\hline Cloning primers & $\begin{array}{l}\text { Bsal site }\left(5^{\prime}-\mathrm{GGTCTC}(\mathrm{N})_{6}-3^{\prime}\right) \\
\text { BamHI site }\left(5^{\prime}-\mathrm{GGATCC}-3^{\prime}\right)\end{array}$ & Notl site $\left(5^{\prime}\right.$-GCGGCCGC- $\left.3^{\prime}\right)$ \\
\hline Full-length clathrin & $\begin{array}{l}\text { 5'-GGG GTC TCG GAT CCG CCC AGA TTC TGC CAA TTC } \\
\text { GTT TTC AGG-3' }\end{array}$ & $\begin{array}{l}5^{\prime} \text {-GCG GCC GCC ATG CTG TAC CCA AAG CCA } \\
\text { GGC TG-3' }\end{array}$ \\
\hline C-terminal clathrin & $\begin{array}{l}5^{\prime} \text {-GGA TCC AAA TTT GAT GTC AAT ACT TCA GCA GTG } \\
\text { CAG GTC-3' }\end{array}$ & $\begin{array}{l}5^{\prime} \text {-GCG GCC GCC ATG CTG TAC CCA AAG CCA } \\
\text { GGC TG-3' }\end{array}$ \\
\hline N-terminal clathrin & $\begin{array}{l}\text { 5'-GGG GTC TCG GAT CCG CCC AGA TTC TGC CAA TTC } \\
\text { GTT TTC AGG-3' }\end{array}$ & $\begin{array}{l}5^{\prime} \text {-GCG GCC GCG TAT ACA CTA AGT GCC AAT } \\
\text { GTC GGG TCC-3' }\end{array}$ \\
\hline
\end{tabular}

Bsal - Notl for N-terminal clathrin (Table 1, cloning primers). Both constructs were cloned into pCR4-TOPO (Invitrogen, Paisley, UK), and positive clones were sequenced (Baseclear). $\mathrm{N}$ - and $\mathrm{C}$-terminal $\mathrm{CHC}$ constructs were then ligated into pUPE (U-Protein Express BV, Utrecht, The Netherlands) containing the Superglo GFP sequence followed by a His-tag at the N-terminus of the C-terminal construct and at the $\mathrm{C}$-terminus of the $\mathrm{N}$-terminal construct. Recombinant $\mathrm{CHC}$ constructs were expressed in HEK293E cells. HEK293E cells from a 1-I suspension culture were pelleted by centrifugation and resuspended in $0.5 \mathrm{M}$ Tris- $\mathrm{HCl}, \mathrm{pH} 7.5$ containing protease inhibitor (Roche), lysed by repeated freeze-thawing and further homogenized in a Potter-Elvehjem homogenizer. Nuclei, organelles, and large protein complexes were removed by sequential centrifugation steps at $1000 \mathrm{~g}$ for $20 \mathrm{~min}, 20000 \mathrm{~g}$ for $20 \mathrm{~min}$, and finally $200000 \mathrm{~g}$ for $1.5 \mathrm{~h}$. Protein constructs were purified from the resulting supernatant by $\mathrm{Ni}^{2+}$-affinity chromatography (Ni Sepharose FF, GE Healthcare Europe $\mathrm{GmbH}$, Diegem, Belgium). Efficient elution from the $\mathrm{Ni}$ Sepharose column was achieved using $25 \mathrm{mM}$ Tris- $\mathrm{HCl}$ (pH 8.5), $500 \mathrm{mM} \mathrm{NaCl}, 500 \mathrm{mM}$ imidazole for C-terminal, and $25 \mathrm{mM}$ Tris- $\mathrm{HCl}$ (pH 7.5), $100 \mathrm{mM}$ EDTA for $\mathrm{N}$-terminal clathrin protein constructs. Eluted proteins were subsequently subjected to gel filtration (Superdex 200 pg 16/ 600; GE Healthcare) and concentrated in microinjection buffer (120 mM KCl, 20 mM HEPES-KOH, pH 6.9; adjusted from Amano et al. (2004)) using Vivaspin tubes with a 10-kDa molecular weight cut-off (GE Healthcare). The yields of $\mathrm{N}$ - and C-terminal $\mathrm{CHC}$ constructs were $\sim 1 \mathrm{mg} / \mathrm{ml}$ protein in 2 and $0.8 \mathrm{ml}$ of microinjection buffer respectively. GFP (Superglo GFP; Qbiogene, Montreal, Quebec, Canada) was dialyzed in the same microinjection buffer and used as negative control. Supplementary Figure 2 (see section on supplementary data given at the end of this article) shows control GFP and purified $\mathrm{N}$ - and C-terminal clathrin protein constructs separated by SDS-PAGE on a $10 \%$ polyacrylamide gel stained with coomassie.

\section{Oocyte isolation, selection, microinjection and culture}

All chemicals were purchased from Sigma Chemical Co., unless otherwise indicated. Cumulus-oocyte complexes (COCs) were collected by aspiration from 3 to $6 \mathrm{~mm}$ follicles from sow (Sus scrofa) ovaries, which were acquired from a slaughterhouse. The collected COCs were allowed to settle and washed several times in a buffer containing $114 \mathrm{mM} \mathrm{NaCl}$, $3.2 \mathrm{mM} \mathrm{KCl}, 2 \mathrm{mM} \mathrm{NaHCO}, 0.4 \mathrm{mM} \mathrm{NaH}{ }_{2} \mathrm{PO}_{4} \cdot \mathrm{H}_{2} \mathrm{O}$,
$0.25 \mathrm{mM}$ sodium pyruvate, $10 \mathrm{mM}$ sodium lactate, $0.5 \mathrm{mM}$ $\mathrm{MgCl}_{2} \cdot 6 \mathrm{H}_{2} \mathrm{O}, 2 \mathrm{mM} \mathrm{CaCl}{ }_{2} \cdot 2 \mathrm{H}_{2} \mathrm{O}, 10 \mathrm{mM}$ HEPES, $0.1 \%(\mathrm{w} / \mathrm{v})$ polyvinylpyrrolidone (PVP), 1 mM dibutyryl cAMP (dbcAMP), $\mathrm{pH}$ 7.4. Oocytes were maintained in prophase arrest during collection and microinjection by the presence of dbcAMP. Prior to injection, oocytes were partially denuded by gentle pipetting until 3-6 layers of cumulus remained, and washed in HEPES-buffered M199 from Gibco BRL (Grand Island, NY, USA) supplemented with $1 \mathrm{mM}$ dbcAMP. For injection, groups of five oocytes were placed in $5 \mu \mathrm{l}$ drops of this medium under oil at $37^{\circ} \mathrm{C}$ on an IX71 inverted microscope (Olympus, Zoeterwoude, The Netherlands) equipped with a heated stage and an epifluorescence setup. A suction pipette was used to immobilize oocytes, and beveled rigid borosilicate micropipettes with a $30^{\circ}$ angle and a $3.5 \mu \mathrm{m}$ tip diameter (custom tips; Eppendorf, Hamburg, Germany) were used to perform injections. Injection solutions were centrifuged for $10 \mathrm{~min}$ at $16000 \mathrm{~g}$ just prior to use, and $\sim 1 \mu \mathrm{l}$ was backloaded into the micropipettes, which were subsequently connected to a Femtojet pressure injection system (Eppendorf). Immediately after injection, epifluorescent images were recorded on the IX71 microscope at $100 \times$ magnification through a FITC filter using a DP20 camera (Olympus) at $100 \mathrm{~ms}$ exposure. Any oocytes showing much higher or lower fluorescence intensity than average (typically $20-30 \%$ of injected oocytes) were immediately removed from the experiment (see for example Fig. 2B). To ensure that equal amounts had been injected between groups, the epifluorescence images taken during injection experiments were evaluated by measuring the total GFP fluorescence per oocyte in ImageJ. Total fluorescence was measured in regions of interest (ROIs) around injected oocytes. ROls were generated using a fixed threshold, which was kept identical between experiments. In vitro maturation was performed as described previously (Hölzenspies et al. 2009).

\section{Immunofluorescence and confocal microscopy}

COCs were washed and denuded by gentle pipetting in $80 \mathrm{mM}$ PIPES, $5 \mathrm{mM}$ EGTA, $2 \mathrm{mM} \mathrm{MgCl} 2,0.3 \%$ (w/v) PVP, pH 6.9 at $37^{\circ} \mathrm{C}$ (PEM-PVP), and then fixed in PEM-PVP containing $4 \%$ ( $\mathrm{v} / \mathrm{v})$ paraformaldehyde (Electron Microscopy Sciences, Hatfield, PA, USA) at room temperature for $1 \mathrm{~h}$. After fixation, oocytes were washed twice in $0.1 \mathrm{M}$ phosphate buffer $(\mathrm{pH} 7.4)$ containing $0.3 \%(\mathrm{w} / \mathrm{v}) \mathrm{PVP}$, and permeabilized in $0.1 \mathrm{M}$ phosphate buffer supplemented with $0.1 \%(\mathrm{w} / \mathrm{v})$ saponin (Riedel de Haen AG, Seelze, Germany). Permeabilized oocytes were blocked in $0.1 \mathrm{M}$ phosphate buffer containing $0.1 \%(\mathrm{w} / \mathrm{v})$ 
saponin, $1 \%(\mathrm{w} / \mathrm{v}) \mathrm{BSA}$ and $2 \%(\mathrm{v} / \mathrm{v})$ normal goat serum (Vector Lab, Burlingame, CA, USA) supplemented with $100 \mathrm{mM}$ glycine for $2 \mathrm{~h}$ at room temperature. After this blocking step, oocytes were subjected to sequential $1 \mathrm{~h}$ incubations with primary and secondary antibodies, which had been diluted in blocking solution without glycine and centrifuged at $100000 \mathrm{~g}$ for $1 \mathrm{~h}$ before use. To label microtubules, a mixture of rabbit polyclonal antibodies directed against $\alpha$-tubulin (1:400; Abcam, Cambridge, UK) and $\beta$-tubulin (1:400; Sigma) was used. Clathrin was labeled with CON.1, which is directed against a conserved region (AA 23-44) of CLC (Nathke et al. 1992), and X22, which is directed against a conserved region (AA 1109-1128) near the C-terminus of CHC (Brodsky 1985, Liu et al. 1995). CON.1 and X22 were produced from hybridoma cultures in our lab (kindly provided by Dr F M Brodsky). Irrelevant primary antibodies of the same isotype (Sigma) and at identical concentrations were used as negative controls. Secondary antibodies were goat anti-mouse IgG alexa488 and goat anti-rabbit IgG alexa568 (Molecular Probes, Eugene, OR, USA), and DNA was labeled for $20 \mathrm{~min}$ with $10 \mu \mathrm{M}$ TO-PRO-3 iodide (Molecular Probes). Each labeling step was followed by three washes in $0.1 \mathrm{M}$ phosphate buffer supplemented with $0.1 \%(\mathrm{w} / \mathrm{v})$ saponin. Finally, oocytes were sealed in Vectashield (Vector Lab) on Superfrost Plus microscope slides (Menzel, Braunschweig, Germany) in $0.12 \mathrm{~mm}$ Secure-Seal Spacers (Molecular Probes).

Confocal images were obtained using a Nikon eclipse TE300 microscope (Nikon Corp., Tokyo, Japan) equipped with a $40 \times$ oil immersion objective (N.A. 1.3) and a Bio-Rad Radiance 2100MP confocal system (Zeiss/Bio-Rad, Hertfordshire, UK). Multichannel images were recorded by sequential excitation using 488-, 543-, and 637-nm lasers. Images were acquired using settings adjusted for submaximal confocal settings (at least $50 \%$ of extracellular pixel values below threshold value and $0.1-0.5 \%$ of pixel values inside the oocyte above maximal pixel value), and analysed using ImageJ (NIH; http://rsb.info. nih.gov/ij/). Submaximal confocal settings were also used to measure spindle recruitment since variations in spindle location and orientation cause large differences in diffraction due to the large size and high lipid content of oocytes (Supplementary Movie 5, see section on supplementary data given at the end of this article). Spindle recruitment was determined by dividing GFP fluorescence intensity on meiotic spindles by that in cortical regions as indicated. Images shown are maximum intensity $\mathrm{Z}$ projections of six consecutive confocal sections (unless otherwise indicated) taken at $2 \mu \mathrm{m}$ intervals and subjected to limited contrast/brightness enhancements up to $\sim 20 \%$. Control images were produced with the highest settings and enhancements used to create images of specific antibody stainings from the same experiment. Supplementary Movies were produced using ImageJ and the VolumeJ plugin (Abramoff \& Viergever 2002) as described in the supplementary data.

\section{Statistical analysis}

Student's $t$-tests and $\chi^{2}$ tests were performed in Microsoft Excel, and multinomial regression was performed in the $\mathrm{R}$ environment (www.r-project.org) using the 'Imer' function of the Ime4 package ( $R$ package version 0.999375-28, http://lme4.r-forge. r-project.org). $P$ values were considered significant at $P<0.05$ (Bonferroni corrected). Mean percentages and S.E.M. were weighted by the number of oocytes in each experiment.

\section{Supplementary data}

This is linked to the online version of the paper at http://dx.doi. org/10.1530/REP-10-0045.

\section{Declaration of interest}

The authors declare that there is no conflict of interest that could be perceived as prejudicing the impartiality of the research reported.

\section{Funding}

This research did not receive any specific grant from any funding agency in the public, commercial, or not-for-profit sector.

\section{Acknowledgements}

We thank the people at the Department of Farm Animal Health for their help with collecting oocytes, P J S van Kooten for production and isolation of CON.1 and X22 antibodies, A de Graaff and R Wubbolts at the Centre for Cellular Imaging for their technical assistance, L C Penning for his help with primer development, E M van $\mathrm{t}$ Veld for her practical contributions to this work and $\mathrm{H}$ Vernooij for statistical advice. We are grateful to A de Vreeden and D R Gutknecht at the University Medical Center for their assistance with and helpful comments on the microinjection procedure.

\section{References}

Abramoff MD \& Viergever MA 2002 Computation and visualization of three-dimensional soft tissue motion in the orbit. IEEE Transactions on Medical Imaging 21 296-304.

Acton SL \& Brodsky FM 1990 Predominance of clathrin light chain LCb correlates with the presence of a regulated secretory pathway. Journal of Cell Biology 111 1419-1426.

Albertson R, Riggs B \& Sullivan W 2005 Membrane traffic: a driving force in cytokinesis. Trends in Cell Biology 15 92-101.

Amano T, Mori T \& Watanabe T 2004 Activation and development of porcine oocytes matured in vitro following injection of inositol 1,4,5trisphosphate. Animal Reproduction Science 80 101-112.

Bjerregaard B \& Maddox-Hyttel P 2004 Regulation of ribosomal RNA gene expression in porcine oocytes. Animal Reproduction Science 82-83 605-616.

Borlido J, Veltri G, Jackson AP \& Mills IG 2008 Clathrin is spindleassociated but not essential for mitosis. PLOS ONE 3 e3115.

Bouniol-Baly C, Hamraoui L, Guibert J, Beaujean N, Szollosi MS \& Debey P 1999 Differential transcriptional activity associated with chromatin configuration in fully grown mouse germinal vesicle oocytes. Biology of Reproduction 60 580-587.

Brodsky FM 1985 Clathrin structure characterized with monoclonal antibodies. I. Analysis of multiple antigenic sites. Journal of Cell Biology 101 2047-2054. 
Cayrol C, Cougoule C \& Wright M 2002 The beta2-adaptin clathrin adaptor interacts with the mitotic checkpoint kinase BubR1. Biochemical and Biophysical Research Communications 298 720-730.

De La Fuente R \& Eppig J 2001 Transcriptional activity of the mouse oocyte genome: companion granulosa cells modulate transcription and chromatin remodeling. Developmental Biology 229 224-236.

DiLuigi A, Weitzman VN, Pace MC, Siano LJ, Maier D \& Mehlmann LM 2008 Meiotic arrest in human oocytes is maintained by a Gs signaling pathway. Biology of Reproduction 78 667-672.

El-Jouni W, Haun S, Hodeify R, Hosein WA \& Machaca K 2007 Vesicular traffic at the cell membrane regulates oocyte meiotic arrest. Development 134 3307-3315.

Fair T, Hyttel P, Greve T \& Boland M 1996 Nucleus structure and transcriptional activity in relation to oocyte diameter in cattle. Molecular Reproduction and Development 43 503-512.

Feng B, Schwarz H \& Jesuthasan S 2002 Furrow-specific endocytosis during cytokinesis of zebrafish blastomeres. Experimental Cell Research 279 14-20.

Goud B, Huet C \& Louvard D 1985 Assembled and unassembled pools of clathrin: a quantitative study using an enzyme immunoassay. Journal of Cell Biology 100 521-527.

Hinckley M, Vaccari S, Horner K, Chen R \& Conti M 2005 The G-proteincoupled receptors GPR3 and GPR12 are involved in CAMP signaling and maintenance of meiotic arrest in rodent oocytes. Developmental Biology 287 249-261.

Hölzenspies JJ, Stoorvogel W, Colenbrander B, Roelen BA, Gutknecht DR \& van Haeften T 2009 CDC2/SPDY transiently associates with endoplasmic reticulum exit sites during oocyte maturation. BMC Developmental Biology 98.

Jones KT 2008 Meiosis in oocytes: predisposition to aneuploidy and its increased incidence with age. Human Reproduction Update 14 143-158.

Kirchhausen T 2000 Clathrin. Annual Review of Biochemistry 69 699-727.

Lee GM 1989 Measurement of volume injected into individual cells by quantitative fluorescence microscopy. Journal of Cell Science $\mathbf{9 4}$ 443-447.

Lehtonen S, Shah M, Nielsen R, lino N, Ryan JJ, Zhou H \& Farquhar MG 2008 The endocytic adaptor protein ARH associates with motor and centrosomal proteins and is involved in centrosome assembly and cytokinesis. Molecular and Cellular Biology $192949-2961$.

Lince-Faria M, Maffini S, Orr B, Ding Y, Claudia F, Sunkel CE, Tavares A, Johansen J, Johansen KM \& Maiato H 2009 Spatiotemporal control of mitosis by the conserved spindle matrix protein Megator. Journal of Cell Biology 184 647-657.

Liu SH, Wong ML, Craik CS \& Brodsky FM 1995 Regulation of clathrin assembly and trimerization defined using recombinant triskelion hubs. Cell 83 257-267.

Liu SH, Marks MS \& Brodsky FM 1998 A dominant-negative clathrin mutant differentially affects trafficking of molecules with distinct sorting motifs in the class II major histocompatibility complex (MHC) pathway. Journal of Cell Biology 140 1023-1037.

Maro B, Johnson MH, Pickering SJ \& Louvard D 1985 Changes in the distribution of membranous organelles during mouse early development. Journal of Embryology and Experimental Morphology 90 287-309.

Martin RH 2008 Meiotic errors in human oogenesis and spermatogenesis. Reproductive Biomedicine Online 16 523-531.

Mehlmann LM 2005a Oocyte-specific expression of Gpr3 is required for the maintenance of meiotic arrest in mouse oocytes. Developmental Biology 288 397-404.
Mehlmann LM 2005b Stops and starts in mammalian oocytes: recent advances in understanding the regulation of meiotic arrest and oocyte maturation. Reproduction 130 791-799.

Mehlmann LM, Saeki Y, Tanaka S, Brennan TJ, Evsikov AV, Pendola FL, Knowles BB, Eppig JJ \& Jaffe LA 2004 The Gs-linked receptor GPR3 maintains meiotic arrest in mammalian oocytes. Science 306 1947-1950.

Montagnac G \& Chavrier P 2008 Endosome positioning during cytokinesis. Biochemical Society Transactions 36 442-443.

Montagnac G, Echard A \& Chavrier P 2008 Endocytic traffic in animal cell cytokinesis. Current Opinion in Cell Biology 20 454-461.

Moore CA, Milano SK \& Benovic JL 2007 Regulation of receptor trafficking by GRKs and arrestins. Annual Review of Physiology 69 451-482.

Motlik J, Kopecny V, Travnik P \& Pivko J 1984 RNA synthesis in pig follicular oocytes. Autoradiographic and cytochemical study. Biology of the Cell $\mathbf{5 0} 229-235$.

Nathke IS, Heuser J, Lupas A, Stock J, Turck CW \& Brodsky FM 1992 Folding and trimerization of clathrin subunits at the triskelion hub. Cell 68 899-910.

Niswonger ML \& O'Halloran TJ 1997 A novel role for clathrin in cytokinesis. PNAS 94 8575-8578.

Ohashi S, Naito K, Liu J, Sheng Y, Yamanouchi K \& Tojo H 2001 Expression of exogenous proteins in porcine maturing oocytes after mRNA injection: kinetic analysis and oocyte selection using EGFP mRNA. Journal of Reproduction and Development 47 351-357.

Okamoto CT, McKinney J \& Jeng YY 2000 Clathrin in mitotic spindles. American Journal of Physiology. Cell Physiology 279 C369-C374.

Richard FJ 2007 Regulation of meiotic maturation. Journal of Animal Science 85 E4-E6.

Royle SJ \& Lagnado L 2006 Trimerisation is important for the function of clathrin at the mitotic spindle. Journal of Cell Science 119 4071-4078.

Royle SJ, Bright NA \& Lagnado L 2005 Clathrin is required for the function of the mitotic spindle. Nature 434 1152-1157.

Schweitzer JK, Burke EE, Goodson HV \& Souza-Schorey C 2005 Endocytosis resumes during late mitosis and is required for cytokinesis. Journal of Biological Chemistry 280 41628-41635.

Ungewickell EJ \& Hinrichsen L 2007 Endocytosis: clathrin-mediated membrane budding. Current Opinion in Cell Biology 19 417-425.

Wang J \& Liu XJ 2003 A G protein-coupled receptor kinase induces Xenopus oocyte maturation. Journal of Biological Chemistry $\mathbf{2 7 8}$ 15809-15814.

Warner AK, Keen JH \& Wang YL 2006 Dynamics of membrane clathrincoated structures during cytokinesis. Traffic 7 205-215.

Wettey FR, Hawkins SF, Stewart A, Luzio JP, Howard JC \& Jackson AP 2002 Controlled elimination of clathrin heavy-chain expression in DT40 lymphocytes. Science 297 1521-1525.

Yamauchi T, Ishidao T, Nomura T, Shinagawa T, Tanaka Y, Yonemura S \& Ishii S 2008 A B-Myb complex containing clathrin and filamin is required for mitotic spindle function. EMBO Journal 27 1852-1862.

Received 25 January 2010

First decision 23 February 2010

Revised manuscript received 17 May 2010

Accepted 2 June 2010 